\title{
Concepções de saúde na formação inicial em educação física: uma perspectiva discente
}

\author{
Itamárcia Oliveira de Melo \\ Graduada em Educação Física - UECE \\ Mestre em Educação - UECE \\ Integrante do Grupo de Estudos e Pesquisa em Educação Física Escolar - GEPEFE/UECE \\ $\triangle$ itamarciaom@gmail.com \\ Heraldo Simões Ferreira \\ Graduado em Educação Física - UNIFOR \\ Doutor em Saúde Coletiva - UECE \\ Professor Adjunto do Curso de Educação Física - UECE \\ Coordenador do Grupo de Estudos e Pesquisa em Educação Física Escolar - GEPEFE/UECE \\ Diogo Queiroz Allen Palácio \\ Graduado em Educação Física - UECE \\ Mestre em Ensino da Saúde - UECE \\ Docente da Rede Estadual de Ensino do Ceará - SEDUC \\ Integrante do Grupo de Estudos e Pesquisa em Educação Física Escolar - GEPEFE/UECE

\section{Karlla da Conceição Bezerra Brito Veras} \\ Graduada em Enfermagem - UNIFOR \\ Mestre em Ensino da Saúde - UECE

\section{Kessiane Fernandes Nascimento} \\ Mestre em Educação -UECE \\ Docente da rede de ensino municipal de Fortaleza \\ Docente do Curso de Educação Física da Faculdade Terra Nordeste - FATENE \\ Integrante do Grupo de Estudos e Pesquisa em Educação Física Escolar - GEPEFE/UECE \\ Stella Lopes Soares \\ Graduada em Educação Física - UVA \\ Graduada em Pedagogia - UNINTA \\ Mestre em Ensino da Saúde - UECE \\ Professora do Curso de Educação Física do Centro Universitário INTA - UNINTA \\ Integrante do Grupo de Estudos e Pesquisa em Educação Física Escolar - GEPEFE/UECE
}

Recebido em 18 de agosto de 2020

Aceito em 28 de setembro de 2020

\section{Resumo:}

O objetivo deste artigo é identificar o conceito de saúde, a partir do olhar dos discentes do Curso de Licenciatura emEducação Física de uma instituição de ensino superior pública. Partindo de umestudo de natureza descritiva comabordagem predominantemente qualitativa, a amostra compreendeu um total de 19 sujeitos com idades entre 23 e 48 anos, sendo 14 homens e 5 mulheres. Os participantes responderam um questionário misto composto por cinco (5) questões. Os resultados da pesquisa revelaram que os aspectos bio/fisiológicos são características essenciais para que uma pessoa seja considerada saudável. Os participantes evidenciaram que durante a sua formação acadêmica tiveram acesso a diversos espaços e recursos educativos de como abordar a saúde na Educação Física Escolar. Para além dos ensinamentos adquiridos no em sala de aula participaram de eventos científicos, 
realizaram leituras de livros e/ou artigos e pesquisas em sites disponíveis na internet. No tocante a Educação Física na escola, a mesma emerge como uma forma de contribuir efetivamente para a saúde dos acadêmicos. Portanto, faz-se necessário compreender a Educação Física enquanto uma disciplina capaz de educar para a saúde no ambiente escolar, além de sensibilizar os docentes para o ensino e prática da saúde em uma perspectiva ampla, abrangente e interdisciplinar.

Palavras-chave: Concepção de Saúde, Educação Física; Escola, Formação Inicial.

\title{
Conceptions of health in initial formation in physical education: the student perspective
}

\begin{abstract}
:
The objective of this article is to identify the concept of health, from the perspective of the students of the Degree in Physical Education at a public higher education institution. Starting from a descriptive study with a predominantly qualitative approach, the sample comprised a total of 19 subjects aged between 23 and 48 years, 14 men and 5 women. Participants answered a mixed questionnaire consisting of five (5) questions. The results of the research revealed that the bio / physiological aspects are essential characteristics for a person to be considered healthy. Participants showed that during their academic training they had access to various educational spaces and resources on how to approach health in Physical Education at School. In addition to the lessons learned in the classroom, they participated in scientific events, read books and / or articles and research on websites available on the internet. With regard to Physical Education at school, it emerges as a way to contribute effectively to the health of academics. Therefore, it is necessary to understand Physical Education as a discipline capable of educating for health in the school environment, in addition to sensitizing teachers to health teaching and practice in a broad, comprehensive and interdisciplinary perspective.
\end{abstract}

Keywords: Health Conception, Physical Education, School; Initial formation.

\section{Concepciones de salud en la formación inicial en educación física: la perspectiva del alumno}

\begin{abstract}
Resumen:
El propósito de este artículo es identificar el concepto de salud, desde la perspectiva de los estudiantes del Curso de Educación Física de una institución pública de educación superior. Partiendo de un estudio descriptivo con un enfoque predominantemente cualitativo, la muestra comprendió un total de 19 sujetos con edades comprendidas entre 23 y 48 años, 14 hombres y 5 mujeres. Los participantes respondieron un cuestionario mixto que consta de cinco (5) preguntas. Los resultados de la investigación revelaron que los aspectos bio / fisiológicos son características esenciales para queuna persona sea considerada saludable. Los participantes mostraron que durante su capacitación académica tuvieron acceso a varios espacios educativos y recursos sobre cómo abord ar la salud en la Educación Física en la Escuela. Además de las lecciones aprendidas en el aula, participaron en eventos científicos, leyeron libros y / o artículos e investigaron sitios web disponibles en Internet. Con respecto a la educación física en la escuela, surge como una forma de contribuir de manera efectiva a la salud de los académicos. Por lo tanto, es necesario entender la Educación Física como una disciplina capaz de educar para la salud en el entorno escolar, además de sensibilizar a los maes tros sobre la enseñanza y la práctica de la salud en una perspectiva amplia, integral e interdisciplinaria.

Palabras clave: Concepción de Salud, Educación Física, la escuela, Formación Inicial.
\end{abstract}




\section{INTRODUÇÃO}

Ao tratar sobre o tema saúde é preciso ter a noção de que há um campo vasto de intervenção em que atuam profissionais de diferentes formações. Em meio a essa diversidade, encontramos formas distintas para compreender o significado do termo saúde, que não é consensual entre indivíduos de um mesmo período histórico (PELICIONE; PELICIONE, 2007).

A concepção de saúde proposta por Christopher Boorse, na década de 1970, esteve associada à ideia de que essa decorre simplesmente da ausência de enfermidades, logo, as ações que se voltavam para este tema, relacionavam-se à prevenção e ao tratamento de doenças em uma esfera fisiológica (SCLIAR, 2007).

Em 1948, a Organização Mundial da Saúde - OMS - ampliou a discussão definindo saúde como sendo "o estado de completo bem-estar físico, mental e social, e não apenas ausência de doenças" (BRASIL, 1997, p.65). Tal publicação implicou no reconhecimento do direito à saúde e da obrigação do Estado em promover e proteger a população do risco de doença, contudo, seu caráter utópico, estável e individual forneceu subsídio para grande número de críticas.

Entre as limitações relacionadas ao conceito proposto pela OMS, existe uma, relacionada à utopia contida na definição, que nos possibilitou levantar a seguinte questão: quem se encontra nesse perfil, ou seja, em completo bem-estar físico, mental e social? A partir de tal questionamento, encontramos outra crítica que diz respeito ao caráter estável apresentado na conceituação, uma vez que, se reporta ao termo "estado", como se uma vez atingido, fosse naturalmente mantido. Por fim, com relação à subjetividade, relativiza a esfera individual, a avaliação da saúde, com base em valores, crenças e experiências particulares, impedindo assim, a possibilidade de uma avaliação dos diversos fatores que incidem sobre ela (BRASIL, 1997).

As restrições relacionadas ao conceito da OMS resultaram em outra forma de compreender a saúde, que, segundo Minayo (2006), passou a ser entendida a partir de seus múltiplos decisivos e condicionantes, relacionados às condições de vida como: a renda, o meio ambiente, a habitação, a educação, a alimentação, a segurança, entre outros. Além das ações do Estado para a promoção da saúde, o avanço da ciência na descoberta de meios que viabilizem uma vida mais saudável à população, os saberes das diferentes áreas que 
interferem sobre a saúde, e, por fim, ao sistema de crenças e valores que cercam o processo de saúde-doença.

Historicamente no Brasil, o compromisso com o direito à saúde foi efetivado mediante a criação do Sistema Único de Saúde - SUS, pela Constituição de 1988, como materialização das Políticas de Saúde do país, decorrente da articulação das lutas sociais e pela democracia e garantia dos direitos humanos. Logo, é oportuno destacar que a adoção pelo SUS por uma concepção de saúde ampliada, permitiu a busca de estratégias pela promoção da saúde em uma perspectiva intersetorial, uma vez que levou em consideração a interferência de diferentes determinantes sociais.

Nas contribuições de Rocha e Centurião (2007) o debate sobre SUS na formação profissional exige discutir seus princípios doutrinários, ou seja, uma prática que garanta a universalidade, equidade e integralidade da atenção.

Deste modo, na tentativa de tornar a formação dos profissionais de saúde consoante aos princípios e diretrizes do SUS, o Ministério da Saúde e da Educação vem, ao longo dos anos, implementando e apoiando ações na formação acadêmica, destacando-se a homologação das Diretrizes Curriculares Nacionais dos cursos de graduação em saúde (BRASIL, 2017). Cabe frisar que esse documento direciona a elaboração dos currículos, indicando elementos como os princípios que norteiam as competências a serem desenvolvidas pelos profissionais da área. Neste contexto, algumas instituições de ensino superior vêm promovendo mudanças curriculares para aproximar a formação dos futuros profissionais às necessidades do SUS.

Nesta perspectiva, o fortalecimento da atenção primária à saúde como campo de intervenção do profissional de Educação Física trouxe novos desafios para a formação desse profissional. Isso implica dizer que as habilidades e competências para intervir no campo da saúde precisam estar em consonância com o modelo de cuidar da saúde, preconizado pelas Políticas Públicas de Saúde da atenção básica, assim como, em conformidade com os serviços e metodologias de trabalho do SUS (COSTA; GARCIA; NAHAS, 2012; ANDRADE et al., 2014).

Logo, é profícuo assinalar que as pesquisas que abrangem o processo de formação dos profissionais de Educação Física, tem avançado no sentido de compreender como o ensino de saúde nos cursos superiores contribuem para a formação acadêmica dos futuros profissionais 
de Educação Física, com o intuito de analisar as reais possibilidades de sua intervenção na área da saúde (BENEDETTI et al., 2014; PASQUIM, 2010; CASTRO; GONÇALVES, 2009; CHACKONMIKAHIL et al., 2009; BRUGNEROTTO; SIMÕES, 2009; ANJOS; DUARTE, 2009).

Cabe esclarecer que dentre os problemas voltados para a formação do profissional de Educação Física, destacam-se: a concepção biomédica e segmentada de promoção de saúde, o modelo de prescrição individualizada de exercícios físicos para intervenções comunitárias, a baixa carga horária dos poucos componentes curriculares específicos em saúde coletiva, à desarticulação interdisciplinar, a falta de aproximação com os serviços de saúde da atenção primária e, por último, a ausência de uma troca de saberes com os outros cursos da área da saúde, fatores que desfavorecem a formação para uma atuação multiprofissional (BRUGNEROTTO; SIMÕES, 2009; ANJOS; DUARTE, 2009; PASQUIM, 2010; SCABAR et al., 2012; ANDRADE et al., 2014).

Portanto, a contextualização apresentada nesta introdução evidencia que foram encontradas algumas limitações no processo de formação do profissional de Educação Física. Diante deste desafio, propomos como objetivo geral identificar o conceito de saúde sob a ótica dos discentes do Curso de Licenciatura em Educação Física de uma instituição de ensino superior pública.

\section{METODOLOGIA}

A presente pesquisa classifica-se como sendo descritiva com abordagem predominantemente qualitativa. A instituição escolhida foi a Universidade Estadual do Ceará - UECE, com foco no Curso de Licenciatura em Educação Física.

A escolha do cenário se justifica por duas grandes razões: primeiro pela relevância do referido curso estar voltado para a formação de professores, uma vez que possui notório reconhecimento social pela qualidade dos serviços prestados. E, por ser o primeiro curso de Educação Física no Estado do Ceará a ser ofertado por uma instituição pública de ensino, vinculado ao Centro de Ciências da Saúde, ou seja, reconhecido pela instituição como curso relacionado à grande área da saúde. 
Os critérios utilizados para a participação do referida pesquisa consistiu na condição dos participantes serem acadêmicos, matriculados em qualquer uma das disciplinas que compunham os dois últimos semestres da matriz curricular, haja vista que, já teriam passado por todas as disciplinas do curso e provável egresso no semestre de aplicação da pesquisa.

O total de alunos que atendeu a esse perfil, de acordo com as informações disponíveis no site da instituição, na ocasião da pesquisa, somavam 19 pessoas. No entanto, participaram apenas quem se encontrava assistindo aula na UECE, no dia da coleta dos dados e que de certa forma manifestou interesse em participar. A amostra compreendeu um total de 19 sujeitos com idades entre 23 e 48 anos, sendo 14 homens e 5 mulheres.

O Curso de Educação Física da UECE oferta em caráter optativo uma disciplina que trata diretamente da saúde, a saber: "Planejamento e Políticas de Saúde", por se tratar de uma disciplina optativa e por ser o profissional de Educação Física reconhecidamente, um profissional da área da saúde, conforme a Resolução 218/97 do Conselho Nacional de Saúde considerou-se que a concepção que envolve este termo deveria ser construída pelos acadêmicos ao longo do curso, independente de haver uma disciplina específica. Portanto, optou-se por não colocar o cumprimento dos créditos relativos à referida disciplina como critério de inclusão.

A coleta de dados ocorreu por meio da aplicação de um questionário composto por cinco (5) questões mistas. As primeiras questões solicitavam que os participantes citassem as características que julgavam essenciais para que uma pessoa pudesse ser considerada saudável, bem como, os fatores que contribuem para se ter saúde. Na sequência do questionário foi perguntado aos estudantes se no decorrer de sua formação acadêmica recebiam informações sobre como abordar a saúde nas aulas de Educação Física e quais outras fontes de informações subsidiavam o conhecimento que possuíam sobre o assunto. Por fim, a última questão solicitava a opinião quanto à efetividade das contribuições das aulas de Educação Física para a saúde dos acadêmicos.

A aplicação do questionário ocorreu durante um dia, em duas salas de aula em que ocorriam aulas de disciplinas que atendiam ao perfil definido neste estudo. Os professores que ministravam as aulas concederam 20 minutos ao final da aula para a aplicação dos 
questionários. Inicialmente houve uma explicação acerca do objetivo do estudo, o procedimento de coleta dos dados e os aspectos éticos envolvidos.

Os acadêmicos que faziam parte do perfil solicitado e que aceitaram participar voluntariamente assinaram um Termo de Compromisso Livre e Esclarecido. Em seguida, responderam ao questionário, sendo possível solicitar explicações ao pesquisador no momento do preenchimento. Ao final, devolveram os instrumentos respondidos.

Para a análise dos dados recorreu-se a temática em estudo, que consiste em “[...] descobrir os núcleos de sentido que compõem uma comunicação, cuja presença ou frequência signifiquem alguma coisa para o objeto analítico visado" (MINAYO, 2014, p.316).

Vale destacar que a pesquisa está de acordo com as orientações da resolução 466/12 do Conselho Nacional de Saúde - CNS, para tanto, foi submetida e aprovada pelo Comitê de Ética do Instituto Superior de Teologia Aplicada - INTA sob o número do parecer: 2.213.634 e CAAE 71465317.0.000000.8133.

\section{RESULTADOS E DISCUSSÃo}

Os participantes foram solicitados a indicar as características essenciais para que uma pessoa seja considerada saudável. Fica patente, portanto, que as respostas reveladas pelos 19 participantes, 10 indicaram apenas aspectos bio/fisiológicos como: estar com as taxas metabólicas controladas, ser capaz de realizar exercícios físicos regularmente, não fazer uso de medicamentos, não possuir doenças e não estar acima do peso. Outros oito (8), além de aspectos bio/fisiológicos, fizeram menção aos aspectos sociais e individuais afetivos, tais como: ter estabilidade afetiva, ter um bom relacionamento social e familiar, ser capaz de desfrutar de atividades de lazer, ter paciência e controle do estresse. E, apenas um (1) evidenciou que além de aspectos bio/fisiológicos, sociais, individuais e afetivos, a saúde transcende o plano individual e abrange questões de políticas públicas e coletivas.

Dessa forma considera-se limitada a menção somente à segurança pública, haja vista que, outros fatores como: o acesso ao atendimento de saúde de qualidade, saneamento básico, moradia, emprego, alimentação, vestuário e meio ambiente não foram mencionados. 
Ao serem solicitados a apontar os fatores que interferem/contribuem para que uma pessoa possa ter saúde, houve uma maior percepção da influência de fatores mais abrangentes. Dos 19 participantes, sete (7) indicaram fatores associados exclusivamente ao aspecto bio/fisiológico: a prática de exercícios e a alimentação. Outros quatro (4) sujeitos apontaram aspectos individuais e afetivos, como: relações afetivas, estresse e autoestima. E, os oito (8) restantes indicaram elementos externos ao indivíduo que depende de intervenções políticas e que exerce influência sobre a saúde, como: o trabalho, o lazer, a economia, a educação e a cultura.

A partir disso, verifica-se que o conceito de saúde é restrito aos fatores biológicos e orgânicos, exclusivamente por induzir e estabelecer uma relação direta entre a prática de atividades físicas e a obtenção da saúde, como se essa última dependesse da primeira em uma relação causal. Logo, apreende-se que a atividade física é compreendida como fator soberano para a promoção da saúde, concebendo a ideia em relação aos programas de Educação Física e promovendo atividades para melhorar a aptidão física relacionada à saúde e assim reduzir o risco de doenças por si só.

É legítimo, contudo, dar ênfase que ao tratar da saúde pública e coletiva, englobamse outros fatores que condicionam ou mesmo determinam esta aquisição. Desta forma, Ferreira et al (2013) destaca que os fatores políticos, econômicos, sociais, culturais e históricos precisam ser também considerados.

Neste viés, a concepção de saúde relacionada a um caráter multifatorial assegura a necessidade de considerar o meio físico, social e cultural como fatores categóricos em que o indivíduo está imerso e que de certa forma implica na saúde dele, uma vez que ele depende dos meios externos como: a qualidade do ar que respira, da miséria, do consumismo, das condições de emprego e de seu estilo de vida (BRASIL, 1997).

Portanto, considera-se limitada a ideia de que apenas um fator, como a prática de exercícios físicos é capaz de resolver os problemas de saúde pública do país. Neste contexto é importante destacar que os exercícios físicos, praticados sob orientação de profissionais habilitados, ajudam a evitar doenças causadas pelo sedentarismo e promove a saúde nos diferentes aspectos individuais: físico, mental e social. 
Em suma, não se trata de desconsiderar a importância de cada fator, mas de reconhecer as limitações de seu alcance quando se considera uma concepção abrangente de saúde. Nesse viés, a Educação Física realizada na escola, ainda que realizada na perspectiva do melhoramento dos aspectos relacionados ao condicionamento físico, tem suas contribuições e limitações.

Ao questionar os participantes se haviam recebido orientações de como abordar a saúde na Educação Física Escolar no decorrer de sua formação acadêmica, sete (7) responderam que sim e justificaram que as disciplinas, as vivências nos estágios, os projetos de extensão, as pesquisas e a participação em eventos sobre saúde coletiva contribuíram para ampliar os conhecimentos acerca da saúde. Cabe destacar, que dos sete (7) participantes que responderam positivamente, apenas três (3) destacaram que em quase todas as disciplinas o tema "saúde" é tratado com o propósito de elucidar como combater o sedentarismo, a obesidade e promover saúde em crianças. Oito (8) sujeitos consideraram parcial, o conhecimento adquirido ao longo da formação, alegando falta de especificidade voltada para o âmbito da escola, pouca profundidade no assunto quando tratado nas disciplinas curriculares do curso, ausência de temas específicos como saúde da criança e um cunho mais prático do que teórico ao tratar o assunto. E por fim, apenas um sujeito declarou não ter recebido informações sobre o assunto até o presente momento de sua formação e justificou sua resposta alegando que o Curso de Licenciatura em Educação Física era muito voltado ao rendimento e atuação fora da escola devido aos professores.

Como postula Nunes (2016) às pressões do mercado de trabalho exigem a formação de um professor de Educação Física que tenha alinhamento com o discurso voltado para o campo da saúde e com foco nos benefícios da prática de diversas atividades físicas, visando assim, a qualidade de vida. No entanto, esse discurso não encontra eco em documentos oficiais que orientam a Educação Física na escola, como a Base Nacional Comum Curricular, documento normativo de âmbito nacional.

Nas contribuições de Guimarães, Neira e Velardi (2015), mesmo encontrando-se na área de Linguagens nos documentos norteadores da prática pedagógica, o discurso da saúde possui grande afinidade com o componente e não deve ser desconsiderado, todavia, deve ser tratado em uma perspectiva abrangente, pautado no paradigma da Promoção da Saúde, que 
considera saúde como sendo "um jogo dinâmico entre a fisiologia, o ambiente, a cultura e as estratégias de intervenção articuladas pela sociedade" (p. 115).

Considerando o dinamismo atual da produção do conhecimento, associado à progressiva facilitação de seu acesso por diferentes mídias, é possível identificar grande variedade de meios para aprender determinado conteúdo fora da programação curricular formal, como participação em congressos, cursos e palestras disponíveis em ambientes virtuais, entre outros. Deste modo, as diferentes formas de se compreender o papel da saúde na Educação Física Escolar não dependem somente do conhecimento veiculado pelas instituições de ensino superior, sendo possível adquirir outros conhecimentos pela via de variadas fontes.

Quando solicitados a indicarem outras formas de acesso ao conhecimento sobre a saúde, além da formação no curso de graduação, oito (8) deles apontaram a troca de conhecimentos com profissionais de outras áreas, nove (9) afirmaram a contribuição das trocas de conhecimento com colegas de faculdade, seis (6) apontaram a participação em eventos científicos, 12 pontuaram a leitura de livros e/ou artigos e nove (9) apontaram as pesquisas em sites disponíveis na internet.

Embora reconhecendo que a formação inicial que ocorre nos cursos de graduação costuma ser considerada o período de maior aquisição dos conhecimentos profissionais, verifica-se, frente ao exposto que a proposta curricular dos cursos de formação não dá conta de devolver à sociedade um profissional totalmente preparado.

Neste sentido, Gonçalves Júnior et al. (2014) discutem que às mudanças ocorridas nas aulas de Educação Física nas escolas são consolidadas no processo de formação de professores, que por sua vez, reflete nos sentidos e significados que este campo de formação assume socialmente. Para tanto, o discurso da promoção da saúde na escola, ocorre devido ao aumento dos casos de doenças hipocinéticas ter gerado uma demanda de formação com perfis profissionais que correspondam a essas expectativas.

Tendo em vista este objetivo, a Educação Física no currículo escolar deve propiciar o conhecimento sobre as atividades físicas voltadas para o bem-estar e saúde, além de estimular atitudes positivas em relação aos exercícios físicos, oportunizar diferentes práticas de forma regular para que possam ser continuadas após o desligamento da escola e promover assim, a 
liberdade na escolha das atividades físicas relacionadas à saúde (NAHAS, 1997; MIRANDA, 2006; HALLAL et al., 2010). Portanto, não podemos desconsiderar as contribuições da Educação Física Escolar no processo de educação para a saúde, apenas não se delega a ela, a exclusividade da responsabilidade na obtenção da mesma.

Os participantes quando indagados se a Educação Física na escola era capaz de contribuir efetivamente para a saúde dos estudantes, dos 19 sujeitos, 15 responderam afirmativamente, em seguida evidenciaram a forma como ocorre: pelo desenvolvimento motor, pela conscientização acerca de hábitos saudáveis e de aspectos sociais que interferem na saúde, pela prática de atividade física, pela divulgação de informações em diferentes veículos de comunicação, melhorando as relações afetivas. O único sujeito que respondeu que contribui parcialmente justificou que esta disciplina escolar possibilita o entendimento do exercício físico, como algo essencial para a boa saúde, o que nos permite deduzir que a Educação Física é capaz de colaborar para uma conscientização acerca da importância da adoção de um estilo de vida saudável.

De acordo com os discentes, a discussão sobre a temática saúde na Educação Física possui uma ligação histórica com a vertente biomédica; nos estudos de Knuth, Azevedo e Rigo (2007), a Educação Física Escolar muitas vezes é fragilizada em função da limitação teórica de sua compreensão. De acordo com Almeida et al. (2011), os autores nos convida a entender que a grande maioria dos participantes desta referida pesquisa observam a Educação Física como meio de evitar problema de saúde e ainda como possibilidades de socialização e lazer, melhorando dessa forma o condicionamento físico de quem a pratica.

Em outro estudo, Marques e Krug (2008), afirmam que a Educação Física tem como objetivo suprir às necessidades e despertar interesses da sociedade, desenvolvendo saúde e bem-estar, sendo uma importante aliada da qualidade de vida e promoção de saúde dentro e fora do âmbito escolar.

Finalizamos esta escrita refletindo acerca da temática saúde nas aulas de Educação Física, dessa forma entendemos a necessidade de ampliar as discussões sobre o tema de forma mais crítica e reflexiva contribuindo assim com o interesse do público escolar atendido. 


\section{CONSIDERAÇÕES FINAIS}

Os resultados da pesquisa indicaram uma forte ligação da compreensão dos acadêmicos sobre o conceito de saúde com componentes apenas fisiológicos e individuais. Até mesmo, quando citados elementos do contexto social, político, econômico e cultural, ocorreu de forma superficial, revelando uma concepção ainda desvinculada de noções relacionadas ao seu aspecto público e coletivo.

Ante ao exposto, é tempestivo apresentar a partir das questões colocadas pelos discentes à necessidade e a importância de uma formação adequada para contribuir com os docentes a respeito do ensino e prática da saúde em uma perspectiva mais ampla, e interdisciplinar durante as aulas de Educação Física na escola.

Assim vislumbra-se que a Educação Física seja tratada como uma ciência capaz de educar para a saúde no ambiente escolar. Neste sentido, acredita-se que, por fazer parte do campo da saúde, o Curso de Licenciatura em Educação Física deveria atentar para a percepção que os alunos têm com relação ao tema "saúde", pois sua atuação profissional implica nas construções teóricas e práticas elaboradas no decorrer do curso.

\section{REFERÊNCIAS}

ALMEIDA, A.B.; TUCHER, G.; ROCHA, C. A. Q.; PAIXÃO, J. A. PERCEPÇÃO DISCENTE SOBRE A EDUCAÇÃO FÍSICA ESCOLAR E MOTIVOS QUE LEVAM À SUA PRÁTICA. Revista Mackenzie de Educação Física e Esporte - v. 10, n. 2, 2011.

ANJOS, T.C., DUARTE, A.C.G.O. A Educação Física e a estratégia de saúde da família: formação e atuação profissional. Physis: Revista de Saúde Coletiva.v.19, n.4, 2009.

ANDRADE, D. R.; COSTA, E. F.; GARCIA, L. M.; FLORINDO, A. A. Formação do bacharel em educação física frente à situação de saúde no Brasil. In: BENEDETTI, T. R. B.; SILVA, D. A. S.; SILVA, K. S. DA; NASCIMENTO, J. V. (Org). A formação do profissional de Educação Física para o setor saúde. Florianópolis: Postmix, 2014.

BRASIL. Parâmetros curriculares nacionais: meio ambiente e saúde. Vol 9. Secretaria de Educação Fundamental. Brasília: MEC, 1997.

BRASIL. Decreto $\mathbf{n}^{\circ} \mathbf{6 . 2 8 6}$, de 5 de dezembro de 2007. Institui o Programa Saúde na Escola - PSE, e dá outras providências. Diário Oficial da União, 06 de dezembro de 2007. 2007.

BRASIL, Conselho Nacional de Saúde. Resolução №466, 2012.

BRASIL, Resoluçãonº 569, de 8 de dezembro de 2017. Conselho Nacional de Saúde: Brasília, 2017.

BRUGNEROTTO, F., SIMÕES, R. Caracterização dos currículos de formaçã o profissional em educação física: um enfoque sobre saúde. Physis: Revista de Saúde Coletiva, v.19, n.01, p.149-172. 2009.

COSTA, F. F.; GARCIA, L. M. T.;NAHAS, M. V. A Educação Física no Brasil em transição: perspectivas para a promoção da atividade física. Rev. Bras. Ativ. Fis. e Saúde. Pelotas. v: 17, n.1, p. 14-21, fev. 2012. 
FERREIRA, Heraldo Simões; SAMPAIO, José Jackson Coelho. Tendências e abordagens daEducação Física escolar e suas interfaces com a saúde. EFDeportes.com, Revista Digital. Buenos Aires. Ano 18, n. 182, jul. 2013. Disponível em: $<$ www.efdeportes.com>. Acesso em 20 de janeiro de 2017.

GONÇALVES JÚNIOR, Luiz; LEMOS, Fábio; CORRÊA, Denise; TORO, Sérgio. Formación de professores em Educación Física en Brasil: compreensión de estudiantes noveles de la Universidad Federal de San Carlos. Estudios Pedagógicos, v. XI, n. especial, p. 87-103, 2014.

GUIMARÃES, Claudia Cristina Pacífico de Assis; NEIRA, Marcos Garcia; VELARDI, Marília. Reflexões sobre saúde e Educação Física escolar: a visão dos professores. Revista Hipótese, v. 1, n. 4, p. 113-138, Itapetininga, 2015.

HALLAL, Pedro Curl; KNUTH, Alan Goulart; CRUZ, Danielle Keylla Alencar; MALTA, Deborah Carvalho. Prática de atividade física em adolescentes brasileiros. Ciência e Saúde Coletiva, v. 15, n. 2, p. 3035-3042, 2010.

KNUTH, A. G.; AZEVEDO, M. R.; RIGO, L. C. A inserção de temas transversais em saúde nas aulas de Educação Física. Revista Brasileira de Atividade Física \& Saúde, Florianópolis, v. 12, n. 3, p. 73-78, set/dez 2007.

MARQUES, M. N.; KRUG, M. R. Educação física escolar: expectativas, importância e objetivos. Revista Digital - Buenos Aires Ano 13 - N 122 - Julho de 2008.

MINAYO, M.C.S. Saúde como responsabilidade cidadã - Maria Cecília de Souza Minayo. In: A saúde em debate na educação física. Bagrichevsky, M.; Palma, A., Estevão, A.; Da Ros, M. Blumenau: Nova Letra, 2006.

MINAYO, M.C.S. O desafio do conhecimento: pesquisa qualitativa em saúde. 14º ed - São Paulo. Hucitec. 2014.

MIRANDA, Made Júnior. Educação física e saúde na escola. Estudos, Goiânia, v. 33, n. 7/8, p. 643-653, jul./ago. 2006.

NAHAS, M. V.. Educação Física no Ensino Médio: educação para um estilo de vida ativo no terceiro milênio. In: Seminário de Educação Física Escolar/ Escola de Educação Física e Esporte, VI. Anais, p. 17-20, 1997.

NUNES, Mário Luiz Ferrari. O mapa do território do Ensino Superior e da formação em Educação Física: emerge o criador. In.: NEIRA, Marcos Garcia; NUNES, Mário Luiz Ferrari (org.). Mostros ou Heróis? Os currículos que formam professors de Educação Física. 1. ed. São Paulo: Phorte, 2016.

ORGANIZAÇÃO MUNDIAL DE SAÚDE. Otawa Charter for Health Promotion. First International Conference on Health Promotion, Otawa, 21 november 1986. WHO/HPR/HEP/95.1. Disponível em: Acesso em: 18 mar. 2016.

PASQUIM, H.M. A saúde coletiva nos cursos de graduação emeducação física. Saúde e Sociedade, v.19, n.01, 192-200p, 2010.

PELICIONE, M.C.F.; PELICIONE, A.F. Educação e promoção da saúde: uma retrospectiva histórica. Disponível em: $<$ www.saocamilo-sp.br/pdf/mundo_saude/55/02_retrospectiva_historica.pdf>. Acesso em maio de 2016.

ROCHA ,V. M.; CENTURIÃO, C. H. Profissionais da saúde: formação, competência e responsabilidade social. In: FRAGA, A. B.; WACHS, F. (Org.). Educação Física e saúde coletiva: políticas de formação e perspectivas de intervenção / Porto Alegre: Editora da UFRGS, p. 17-32, 2007.

SANTOS JUNIOR, E; W.; CEZAR, E. H. A.; LEITE, S. Q. M.; LIMA, M. C. B "Estudo exploratório sobre a percepção dos sabere s de saúde realizada no Ensino Médio do CEFET de química de Nilópolis/RJ (Brasil)". Anais do XX Congresso Internacional de Educação Física, FIEP Bulletin, vol. 75, Special edition, Foz do Iguaçu, 2005.

SCABAR, T. G.; PELICIONI, A. F.; PELICIONI, M. C. F. Atuação do profissional de Educação Física no Sistema Único de Saúde: uma análise a partir da Política Nacional de Promoção da Saúde e das Diretrizes do Núcleo de Apoio à Saúde da Família - NASF. J. Health Sci. Inst. v. 30, n. 4, 411-8p, 2012.

SCLIAR, Moacyr. História do conceito de saúde. Physis: Revista Saúde Coletiva. Rio de Janeiro, p. 29-41. 2007.

\section{$(\mathrm{cc}) \mathrm{EY}_{\mathrm{B}}$}

Este trabalho está licenciado com uma Licença Creative Commons - Atribuição 4.0 Internacional. 